\section{Las tecnologías de información y comunicación en la formación académica de los estudiantes de pregrado de la Facultad de Ciencias Administrativas - UNMSM 2017}

\author{
Information and communication technologies in the \\ academic training of undergraduate students of the faculty \\ of administrative sciences - UNMSM 2017
}

\section{RESUMEN}

A través del presente trabajo se pretende analizar la interacción de los estudiantes de la Facultad de Ciencias Administrativas, a través de los espacios virtuales, profundizando en sus posibilidades y destacando la necesidad de flexibilizar la educación. La Tecnología de la información y comunicación se ha convertido en un instrumento muy importante en el sistema de enseña universitaria, el uso adecuado de estas, permitirá que los estudiantes tengan mayor facilidad, para su proceso de aprendizaje, la búsqueda avanzada de la información de bases de datos y revistas científicas indexadas. El uso del internet es uno de medios muy importe en el proceso de la formación académico de los estudiantes.

Palabras claves: Tecnología; enseñanza; instrumento; espacios virtuales; educación.

\begin{abstract}
Through this work is intended to analyze the interaction of students of the Faculty of Administrative Sciences, through virtual spaces, deepening their possibilities and highlighting the need to make education more flexible. Information and communication technology has become a very important instrument in the system of university teaching, the proper use of these, will allow students to have greater ease, for their learning process, the advanced search of information bases of data and indexed scientific journals. The use of the internet is one of very important means in the process of the academic formation of the students.
\end{abstract}

Keywords: Technology; teaching; instrument; virtual spaces; education.

\section{Vicente Armas Edgar ${ }^{1}$}

edgarvicente02@gmail.com

Universidad Nacional Mayor de San Marcos, Facultad de Ciencias Administrativas

\footnotetext{
${ }^{1}$ Doctor en Administración, Magíster en Administración con Mención en Gestión Empresarial, Docente asociado de pregrado y posgrado de la Facultad de Ciencias Administrativas de la Universidad Nacional Mayor de San Marcos.
}

(C) Los autores. Este artículo es publicado por Gestión en el Tercer Milenio de la Facultad de Ciencias Administrativas de la Universidad Nacional Mayor de San Marcos. Este es un artículo de acceso abierto, distribuido bajolos términos de la licencia Creative Commons Atribucion - No Comercia_Compartir Igual 4.0 Internacional. (http://creativecommons.org/licenses/by-nc-sa/4.0/) que permite el uso no comercial, distribución y reproducción en cualquier medio, siempre que la obra original sea debidamente citada. 


\section{INTRODUCCIÓN}

Las Tecnologías de la Información y la Comunicación (TIC), en especial los servicios que ofrece Internet, proporciona una enseñanza personalizada y un seguimiento continuo de los progresos del alumnado, optimizando el proceso de aprendizaje y eliminando barreras espacio-temporales. Este entorno nos va a facilitar la comunicación e interacción entre los distintos agentes, configurando así diferentes espacios comunicativos: para la tutoría, para la comunicación social, de soporte en las diferentes situaciones didácticas, así como en las organizativas, entre otras.

La presente investigación tiene como objetivo demostrar si los estudiantes de la Facultad de Ciencias Administrativas vienen haciendo uso adecuado del internet en la interacción con personas, empresas e incluso realizar curso a distancia. A través del buen uso de este tipo de herramientas se puede avanzar hacia una educación competitiva y que además trate de potenciar un aprendizaje y una enseñanza permanente, necesaria en nuestros días. Las redes sociales se han convertido actualmente en una de las herramientas fundamentales de Internet.

\section{MÉTODO}

Para el desarrollo del presente estudio se utilizó el tipo de investigación descriptivo con enfoque cuantitativo. El diseño es no Experimental, transversal. La población objetivo ha sido el total de estudiantes matriculados del Semestre Académico del 2017, que ascienden a 2,366 estudiantes. La muestra ha sido de 100 estudiantes, afijados proporcionalmente según el total de estudiantes de cada Escuela Profesional.

\section{RESULTADOS}

\section{Conexión a internet}

Del total, el 57\% de los encuestados usa internet desde el hogar, el 13\% desde la cabina pública y en un $30 \%$ desde otro lugar. Por Escuelas destaca la Escuela de Administración con $36.8 \%$, seguido con $33.3 \%$ la Escuela de Administración de Negocios Internacionales y finalmente con 29.8\% la Escuela de Administración de Turismo (Tabla 1).

\section{Frecuencia de Conexión a Internet}

Del total encuestado, el 85\% todo el día, 11\% inter diario y $4 \%$ de recién en cuando. Según Escuelas; en el rubro todos los días el 52.9\% de los encuestados corresponde a la Escuela Profesional de Administración, 21.2\% la Escuela de Administración de Turismo y el 25.9\% la Escuela de Administración de Negocios Internacionales (Tabla 2).

\section{Tiempo de Conexión a Internet}

Según tiempo de conexión a internet, del total de encuestado, el $70 \%$ manifiestan estar conectado más de 2 horas, $19 \%$ dos horas y $11 \%$ una hora. Del Total que manifiestan más de dos horas el 62.9\% corresponde a la Escuela Profesional de Administración, 18,6\% respectivamente la Escuela Profesional de Negocios Internacionales y la Escuela Profesional de Turismo (Tabla 3).

Tabla 1.

Lugar de Conexión a Internet por Escuelas Académicas Profesionales dela Facultad de Ciencias Administrativas-UNMSM a noviembre 2017

\begin{tabular}{|c|c|c|c|c|c|c|}
\hline & & & \multicolumn{3}{|c|}{ Escuela Académico Profesional } & \multirow[b]{2}{*}{ Total } \\
\hline & & & Administración & $\begin{array}{c}\text { Negocios } \\
\text { Internacionales }\end{array}$ & Turismo & \\
\hline \multirow{6}{*}{$\begin{array}{l}\text { ¿Desde dónde } \\
\text { te conectas } \\
\text { a internet } \\
\text { mayormente? }\end{array}$} & \multirow{2}{*}{ casa } & Recuento & 21 & 19 & 17 & 57 \\
\hline & & $\%$ & $36,8 \%$ & $33,3 \%$ & $29,8 \%$ & $100,0 \%$ \\
\hline & \multirow{2}{*}{$\begin{array}{l}\text { Cabina de } \\
\text { Internet }\end{array}$} & Recuento & 2 & 5 & 6 & 13 \\
\hline & & $\%$ & $15,4 \%$ & $38,5 \%$ & $46,2 \%$ & $100,0 \%$ \\
\hline & \multirow{2}{*}{ otros } & Recuento & 25 & 4 & 1 & 30 \\
\hline & & $\%$ & $83,3 \%$ & $13,3 \%$ & $3,3 \%$ & $100,0 \%$ \\
\hline \multirow{2}{*}{\multicolumn{2}{|c|}{ Total }} & Recuento & 48 & 28 & 24 & 100 \\
\hline & & $\%$ & $48,0 \%$ & $28,0 \%$ & $24,0 \%$ & $100,0 \%$ \\
\hline
\end{tabular}

Fuente: Elaboración propia 
Tabla 2.

Frecuencia de conexión a internet por Escuelas Académicas Profesionales dela Facultad de Ciencias AdministrativasUNMSM a noviembre 2017

\begin{tabular}{|c|c|c|c|c|c|c|}
\hline & & & \multicolumn{3}{|c|}{ Escuela Académico Profesional } & \multirow{2}{*}{ Total } \\
\hline & & & Administración & $\begin{array}{c}\text { Negocios } \\
\text { Internacionales }\end{array}$ & Turismo & \\
\hline \multirow{6}{*}{$\begin{array}{l}\text { ¿Conque } \\
\text { frecuencia te } \\
\text { conectas a } \\
\text { internet? }\end{array}$} & \multirow{2}{*}{ Todos los días } & Recuento & 45 & 22 & 18 & 85 \\
\hline & & $\%$ & $52.9 \%$ & $25.9 \%$ & $21.2 \%$ & $100.0 \%$ \\
\hline & \multirow{2}{*}{ Inter diario } & Recuento & 2 & 3 & 6 & 11 \\
\hline & & $\%$ & $18.2 \%$ & $27.3 \%$ & $54.5 \%$ & $100.0 \%$ \\
\hline & \multirow{2}{*}{$\begin{array}{l}\text { Una vez por } \\
\text { semana }\end{array}$} & Recuento & & 3 & 0 & 4 \\
\hline & & $\%$ & $25.0 \%$ & $75.0 \%$ & $0.0 \%$ & $100.0 \%$ \\
\hline \multirow{2}{*}{\multicolumn{2}{|c|}{ Total }} & Recuento & 48 & 28 & 24 & 100 \\
\hline & & $\%$ & $48.0 \%$ & $28.0 \%$ & $24.0 \%$ & $100.0 \%$ \\
\hline
\end{tabular}

Fuente: Elaboración propia

Tabla 3

Tiempo de conexión a internet por Escuelas Académicas Profesionales dela Facultad de Ciencias Administrativas-UNMSM a noviembre 2017

\begin{tabular}{|c|c|c|c|c|c|c|}
\hline & & & \multicolumn{3}{|c|}{ Escuela Académico Profesional } & \multirow[b]{2}{*}{ Total } \\
\hline & & & Administración & $\begin{array}{c}\text { Negocios } \\
\text { Internacionales }\end{array}$ & Turismo & \\
\hline \multirow{6}{*}{$\begin{array}{l}\text { ¿Cuánto tiempo } \\
\text { permanece } \\
\text { conectado a } \\
\text { internet' }\end{array}$} & \multirow{2}{*}{ Una hora } & Recuento & 0 & 10 & 1 & 11 \\
\hline & & $\%$ & $0.0 \%$ & $90.9 \%$ & $9.1 \%$ & $100.0 \%$ \\
\hline & \multirow{2}{*}{ dos horas } & Recuento & 4 & 5 & 10 & 19 \\
\hline & & $\%$ & $21.1 \%$ & $26.3 \%$ & $52.6 \%$ & $100.0 \%$ \\
\hline & \multirow{2}{*}{$\begin{array}{c}\text { más de dos } \\
\text { horas }\end{array}$} & Recuento & 44 & 13 & 13 & 70 \\
\hline & & $\%$ & $62.9 \%$ & $18.6 \%$ & $18.6 \%$ & $100.0 \%$ \\
\hline \multirow{2}{*}{\multicolumn{2}{|c|}{ Total }} & Recuento & 48 & 28 & 24 & 100 \\
\hline & & $\%$ & $48.0 \%$ & $28.0 \%$ & $24.0 \%$ & $100.0 \%$ \\
\hline
\end{tabular}

Fuente: Elaboración propia

\section{Uso del correo electrónico}

Del total encuestado, el $41 \%$ manifiestan de qué casi siempre usan el correo electrónico, 38\% a veces el $11 \%$ siempre, $8 \%$ casi nunca y el $2 \%$ nunca.

De los que han manifestado casi siempre el uso del correo el $53.7 \%$ corresponden a los encuestados en la Escuela Profesional de Administración, 22\% a la Escuela Profesional de Turismo y el $24,3 \%$ a la Escuela Profesional de Negocios Internacionales (Tabla 4).

\section{Uso de Redes Sociales}

Del total encuestado, el 62\% ha manifestado de que siempre hace uso de las redes sociales, 33\% casi siempre y el $5 \%$ a veces.

De los que manifiestan siempre hacen uso de las redes sociales por escuelas, el $48.4 \%$ de los encuestados pertenecen a la Escuela Profesional de Administración, 30,6\% a la Escuela Profesional de Administración de Turismo y el $21 \%$ a la Escuela Profesional de Negocios Internacionales (Tabla 5).

\section{Uso Educativo-Tutorial}

Del total, el 49\% de los entrevistados indicaron, de que casi siempre usan tutoriales para aprender un tema académico, el $28 \%$ a veces, $11 \%$ siempre y $12 \%$ casi nunca.

De los que manifestaron casi siempre usan el tutorial con fines académicos, el $46.9 \%$ corresponde a la Escuela Profesional de Administración, $30.6 \%$ a la Escuela Profesional de Negocios Internacionales y el $22.4 \%$ a la Escuela Profesional de Administración de Turismo (Tabla 6). 
Tabla 4

Uso del Correo Electrónico por Escuelas Académicas Profesionales de la Facultad de Ciencias Administrativas-UNMSM noviembre 2017

\begin{tabular}{|c|c|c|c|c|c|c|}
\hline & \multicolumn{3}{|c|}{ Escuela Académico Profesional } & \multirow[b]{2}{*}{ Total } \\
\hline & & & Administración & \begin{tabular}{c|} 
Negocios \\
Internacionales
\end{tabular} & Turismo & \\
\hline \multirow{10}{*}{$\begin{array}{l}\text { Reviso mi correo } \\
\text { electrónico }\end{array}$} & \multirow{2}{*}{ Nunca } & Recuento & 2 & 0 & 0 & 2 \\
\hline & & $\%$ & $100.0 \%$ & $0.0 \%$ & $0.0 \%$ & $100.0 \%$ \\
\hline & \multirow{2}{*}{ Casi nunca } & Recuento & 4 & 2 & 2 & 8 \\
\hline & & $\%$ & $50.0 \%$ & $25.0 \%$ & $25.0 \%$ & $100.0 \%$ \\
\hline & \multirow{2}{*}{ A veces } & Recuento & 16 & 11 & 11 & 38 \\
\hline & & $\%$ & $42.1 \%$ & $28.9 \%$ & $28.9 \%$ & $100.0 \%$ \\
\hline & \multirow{2}{*}{ Casi siempre } & Recuento & 22 & 10 & 9 & 41 \\
\hline & & $\%$ & $53.7 \%$ & $24.4 \%$ & $22.0 \%$ & $100.0 \%$ \\
\hline & \multirow{2}{*}{ Siempre } & Recuento & 4 & 5 & 2 & 11 \\
\hline & & $\%$ & $36.4 \%$ & $45.5 \%$ & $18.2 \%$ & $100.0 \%$ \\
\hline \multirow{2}{*}{\multicolumn{2}{|c|}{ Total }} & Recuento & 48 & 28 & 24 & 100 \\
\hline & & $\%$ & $48.0 \%$ & $28.0 \%$ & $24.0 \%$ & $100.0 \%$ \\
\hline
\end{tabular}

Fuente: Elaboración propia

Tabla 5.

Uso de las Redes Sociales por Escuelas Académicas Profesionales de la Facultad de Ciencias Administrativas-UNMSM a noviembre 2017

\begin{tabular}{|c|c|c|c|c|c|c|}
\hline & & & Escuela & Académico Prof & onal & \\
\hline & & & Administración & \begin{tabular}{c|} 
Negocios \\
Internacionales \\
\end{tabular} & Turismo & Total \\
\hline & A urog & Recuento & 1 & 4 & 0 & 5 \\
\hline Ingreso a mis & A VECES & $\%$ & $20.0 \%$ & $80.0 \%$ & $0.0 \%$ & $100.0 \%$ \\
\hline redes sociales & Cociciomnr & Recuento & 17 & 11 & 5 & 33 \\
\hline instragram, & Casi siempre & $\%$ & $51.5 \%$ & $33.3 \%$ & $15.2 \%$ & $100.0 \%$ \\
\hline twuiter, etc) & Cismor & Recuento & 30 & 13 & 19 & 62 \\
\hline & Siempre & $\%$ & $48.4 \%$ & $21.0 \%$ & $30.6 \%$ & $100.0 \%$ \\
\hline & & Recuento & 48 & 28 & 24 & 100 \\
\hline & & $\%$ & $48.0 \%$ & $28.0 \%$ & $24.0 \%$ & $100.0 \%$ \\
\hline
\end{tabular}

Fuente: Elaboración propia

Tabla 6

Uso Educativo-Tutorial por Escuelas Académicas Profesionales de la Facultad de Ciencias Administrativas-UNMSM a noviembre 2017

\begin{tabular}{|c|c|c|c|c|c|c|}
\hline & \multicolumn{3}{|c|}{ Escuela Académico Profesional } & \multirow[b]{2}{*}{ Total } \\
\hline & & & Administración & $\begin{array}{c}\text { Negocios } \\
\text { Internacionales }\end{array}$ & Turismo & \\
\hline \multirow{8}{*}{$\begin{array}{l}\text { Uso tutoriales } \\
\text { para aprender un } \\
\text { tema }\end{array}$} & \multirow{2}{*}{ Casi nunca } & Recuento & 1 & 9 & 2 & 12 \\
\hline & & $\%$ & $8.3 \%$ & $75.0 \%$ & $16.7 \%$ & $100.0 \%$ \\
\hline & \multirow{2}{*}{ A veces } & Recuento & 18 & 2 & 8 & 28 \\
\hline & & $\%$ & $64.3 \%$ & $7.1 \%$ & $28.6 \%$ & $100.0 \%$ \\
\hline & \multirow{2}{*}{ Casi siempre } & Recuento & 23 & 15 & 11 & 49 \\
\hline & & $\%$ & $46.9 \%$ & $30.6 \%$ & $22.4 \%$ & $100.0 \%$ \\
\hline & \multirow{2}{*}{ Siempre } & Recuento & 6 & 2 & 3 & 11 \\
\hline & & $\%$ & $54.5 \%$ & $18.2 \%$ & $27.3 \%$ & $100.0 \%$ \\
\hline \multirow{2}{*}{\multicolumn{2}{|c|}{ Total }} & Recuento & 48 & 28 & 24 & 100 \\
\hline & & $\%$ & $48.0 \%$ & $28.0 \%$ & $24.0 \%$ & $100.0 \%$ \\
\hline
\end{tabular}

Fuente: Elaboración propia 


\section{Acceso a base de datos electrónicos}

Del Total encuestado 47\% manifiestan de qué casi nunca han hecho uso de las bases de datos electrónicos, $24 \%$ han manifestado nunca han hecho uso, $16 \%$ manifiestan a veces y $13 \%$ casi siempre.

De los que manifiestan casi siempre usa la base de datos electrónicos, $46.2 \%$ corresponde a la Escuela Profesional de Administración, el 38.5\% a la Escuela de Profesional de Administración de Negocios Internacionales y $15.4 \%$ a la Escuela Profesional de Administración de Turismo (Tabla 7).

\section{Tomar cursos en línea}

El 38\% de los encuestados señaló que casi nunca han toma cursos en línea, $29 \%$ nunca, $23 \%$ a veces, $8 \%$ casi siempre y $2 \%$ siempre.

De los manifiestan nunca y casi nunca toman cursos en línea, el $46.5 \%$ corresonden a encuestados que pertenecen a la Escuela Profesional de Administración, 27.4\% a la Escuela de Administración de Negocios Internacionales y el $26.1 \%$ a la Escuela de Administración de Turismo (Tabla 8).

\section{Tabla 7}

Uso de la base de datos electrónico de la universidad (ebesco, proquest, etc) por Escuelas Académicas Profesionales de la Facultad de Ciencias Administrativas-UNMSM a noviembre 2017

\begin{tabular}{|c|c|c|c|c|c|c|}
\hline & & & \multicolumn{3}{|c|}{ Escuela Académico Profesional } & \multirow[b]{2}{*}{ Total } \\
\hline & & & Administración & \begin{tabular}{|c|} 
Negocios \\
Internacionales \\
\end{tabular} & Turismo & \\
\hline \multirow{8}{*}{$\begin{array}{c}\text { Uso de la } \\
\text { base de datos } \\
\text { electrónico de } \\
\text { la universidad } \\
\text { (ebesco, } \\
\text { proquest,etc) }\end{array}$} & \multirow{2}{*}{ Nunca } & Recuento & 4 & 6 & 14 & 24 \\
\hline & & $\%$ & $16.7 \%$ & $25.0 \%$ & $58.3 \%$ & $100.0 \%$ \\
\hline & \multirow{2}{*}{ Casi nunca } & Recuento & 29 & 14 & 4 & 47 \\
\hline & & $\%$ & $61.7 \%$ & $29.8 \%$ & $8.5 \%$ & $100.0 \%$ \\
\hline & \multirow{2}{*}{ A veces } & Recuento & 9 & 3 & 4 & 16 \\
\hline & & $\%$ & $56.3 \%$ & $18.8 \%$ & $25.0 \%$ & $100.0 \%$ \\
\hline & \multirow{2}{*}{ Casi siempre } & Recuento & 6 & 5 & 2 & 13 \\
\hline & & $\%$ & $46.2 \%$ & $38.5 \%$ & $15.4 \%$ & $100.0 \%$ \\
\hline \multirow{2}{*}{\multicolumn{2}{|c|}{ Total }} & Recuento & 48 & 28 & 24 & 100 \\
\hline & & $\%$ & $48.0 \%$ & $28.0 \%$ & $24.0 \%$ & $100.0 \%$ \\
\hline
\end{tabular}

Fuente: Elaboración propia

\section{Tabla 8}

Tomar cursos en línea por Escuelas Académicas Profesionales de la Facultad de Ciencias Administrativas-UNMSM a noviembre 2017

\begin{tabular}{|c|c|c|c|c|c|c|}
\hline & & & \multicolumn{3}{|c|}{ Escuela Académico Profesional } & \multirow[b]{2}{*}{ Total } \\
\hline & & & Administración & \begin{tabular}{c|} 
Negocios \\
Internacionales \\
\end{tabular} & Turismo & \\
\hline \multirow{10}{*}{$\begin{array}{l}\text { Tomo cursos en } \\
\text { linea }\end{array}$} & \multirow{2}{*}{ Nunca } & Recuento & 10 & 5 & 14 & 29 \\
\hline & & $\%$ & $34.5 \%$ & $17.2 \%$ & $48.3 \%$ & $100.0 \%$ \\
\hline & \multirow{2}{*}{ Casi nunca } & Recuento & 20 & 11 & 7 & 38 \\
\hline & & $\%$ & $52.6 \%$ & $28.9 \%$ & $18.4 \%$ & $100.0 \%$ \\
\hline & \multirow{2}{*}{ A veces } & Recuento & 16 & 4 & 3 & 23 \\
\hline & & $\%$ & $69.6 \%$ & $17.4 \%$ & $13.0 \%$ & $100.0 \%$ \\
\hline & \multirow{2}{*}{ Casi siempre } & Recuento & 1 & 7 & 0 & 8 \\
\hline & & $\%$ & $12.5 \%$ & $87.5 \%$ & $0.0 \%$ & $100.0 \%$ \\
\hline & \multirow{2}{*}{ Siempre } & Recuento & 1 & 1 & 0 & 2 \\
\hline & & $\%$ & $50.0 \%$ & $50.0 \%$ & $0.0 \%$ & $100.0 \%$ \\
\hline \multirow{2}{*}{\multicolumn{2}{|c|}{ Total }} & Recuento & 48 & 28 & 24 & 100 \\
\hline & & $\%$ & $48.0 \%$ & $28.0 \%$ & $24.0 \%$ & $100.0 \%$ \\
\hline
\end{tabular}

Fuente: Elaboración propia 


\section{DISCUSIÓN}

La investigación realizada sobre si los estudiantes de la Facultad de Ciencias Administrativas vienen usando adecuadamente la Tecnología de la Información y Comunicación en proceso de su formación académica los resultados se muestran a continuación:

El 57\% de los estudiantes encuestados de la Facultad accede a internet desde su hogar y el restante de otros lugares; el $85 \%$ se interconectan todos los días; el 70\% los hace por más de dos horas; el $52 \%$ hacen uso del correo electrónico siempre y casi siempre; el $62 \%$ hacen uso siempre y casi siempre de las redes sociales.

Con fines educativos; el $60 \%$ de los encuestados manifiestan de que siempre y casi siempre usan el tutorial para repasar temas académicos. Sólo el 13\% de los encuestados manifiestan de que casi siempre hacen uso de la base de datos electrónicos para realizar tareas académicas e investigación y solo el 10\% de los encuestados manifestaron siempre y casi siempre llevan cursos en línea.

\section{CONTRASTACIÓN DE LA HIPOTESIS}

Para contrastación de la hipótesis se ha tomado en consideración aquellas interacciones relacionados con el uso del internet con fines educativos; Tutorial, acceso a base de datos electrónicos y cursos en línea.

$1^{\circ}$ Paso: Formulación de hipótesis estadístico

$\mathrm{H}_{\mathrm{o}}$ : La interacción con fines educativos, en el uso de las TIC, viene contribuyendo equitativamente en el proceso de formación académica de los estudiantes de la Facultad de Ciencias Administrativas.

$\mathrm{H}_{1}$ : La interacción con fines educativos, en el uso de las TIC, viene contribuyendo no equitativamente en el proceso de formación académica de los estudiantes de la Facultad de Ciencias Administrativas

$2^{\circ}$ Paso: Grados de libertad y nivel de significación

Se ha usado el estadístico Chi Cuadrado (3-1) 2 grados de libertad y un nivel de significación del $5 \%$. $3^{\circ}$ Paso Resultado

Se ha procesado con el SPSS siendo el resultado:

\begin{tabular}{|c|c|c|c|}
\hline \multicolumn{4}{|c|}{ Contribución Académica } \\
\hline & N observado & N esperada & Residuo \\
\hline Uso del Tutorial & 60 & 27,7 & 32,3 \\
\hline $\begin{array}{c}\text { Acceso a la Base de } \\
\text { Datos Electrónicos }\end{array}$ & 13 & 27,7 & $-14,7$ \\
\hline Cursos en Línea & 10 & 27,7 & $-17,7$ \\
\hline Total & 83 & & \\
\hline
\end{tabular}

\begin{tabular}{|c|c|}
\hline \multicolumn{2}{|c}{ Estadísticos de prueba } \\
\hline Chi-cuadrado & Contribución Académica \\
\hline gl & $56,843^{\mathrm{a}}$ \\
\hline Sig. asintótica & 2 \\
\hline
\end{tabular}

a. 0 casillas $(0,0 \%)$ han esperado frecuencias menores que 5. La frecuencia mínima de casilla esperada es 27,7.

Dado que el nivel de significación calculado es 0,000 , menor que $\mathrm{P}=0,05$ nivel de significación crítico, con una Chi Cuadrado de 56, 843; por lo tanto, se rechaza $\mathrm{H}_{\mathrm{o}}$ y sé acepta $\mathrm{H}_{1}$, por lo tanto, el uso de las TIC, no viene contribuyendo uniformemente al proceso de formación académica de los estudiantes de la Facultad de Ciencias Administrativas.

\section{CONCLUSIONES}

Se concluye de que con fines educativos los estudiantes encuestados, el $60 \%$ de que siempre y casi siempre usan el tutorial para repasar temas académicos y el $40 \%$ con otros fines; Sólo el 13\% de los encuestados manifiestan de que siempre y casi siempre hacen uso de la base de datos electrónicos para realizar tareas académicas e investigación y el $87 \%$ restante a veces, casi nunca y nunca, este es un resultado importante, hay evidencias en el uso, pero se debe seguir capacitando a docentes y estudiantes en hacer uso de esta herramienta que es muy importante para la investigación y finalmente solo el $10 \%$ de los encuestados manifestaron casi siempre llevan cursos en línea y el $90 \%$ a veces, casi nunca y nunca; la Universidad de San Marcos viene capacitando el uso de las aulas virtuales y también la Facultad los resultados mejorarán en el futuro. 


\section{BIBLIOGRAFÍA}

Chavez Ruiz, Marlon y Chavez Ruiz, Hanny (2008), "Uso de Internet y rendimiento académico de los estudiantes de la FCEH - Universidad Nacional de la Amazonía peruana Iquitos.

Cruz Hinostroza, A. J., Gil Samamé, A. J., \& San Martin Ortiz, A. Y. (2016). Efectos sociales y comunicacionales de la Internet en los estudiantes de la Facultad de Psicología de la UNHEVAL Huánuco 2015. Universidad Nacional Hermilio Valdizán.

Guardia, Sergio (1995). Pronóstico. Definición, importancia y tipos de pronóstico Mexico
Peyrolón, Pablo (2007). Usos lúdicos de internet entre estudiantes universitarios España

Silva, Roberto (1999). El periodismo electrónico y presencia peruana en internet. Tesis de la Universidad Nacional Mayor de San Marcos.

Torres Acuña, W. J. (2003). Utilización de Internet y el bienestar psicológico en estudiantes universitarios de alto y bajo nivel de acceso a la tecnología de información por computadora. Universidad Nacional Mayor de San Marcos. 
